\title{
Self-organization of complex networks as a dynamical system
}

AUTHOR(S):

Aoki, Takaaki; Yawata, Koichiro; Aoyagi, Toshio

\section{CITATION:}

Aoki, Takaaki ... [et al]. Self-organization of complex networks as a dynamical system. Physical review. E, Statistical, nonlinear, and soft matter physics 2015, 91(1): 012908.

ISSUE DATE:

2015-01-12

URL:

http://hdl.handle.net/2433/201831

RIGHT:

C2015 American Physical Society 


\title{
Self-organization of complex networks as a dynamical system
}

\author{
Takaaki Aoki, ${ }^{1, *}$ Koichiro Yawata, ${ }^{2}$ and Toshio Aoyagi ${ }^{2,3}$ \\ ${ }^{1}$ Faculty of Education, Kagawa University, Takamatsu 760-8521, Japan \\ ${ }^{2}$ Graduate School of Informatics, Kyoto University, Kyoto 606-8501, Japan \\ ${ }^{3}$ CREST, Japan Science and Technology Agency, Kawaguchi, Saitama 332-0012, Japan
}

(Received 11 October 2014; published 12 January 2015)

\begin{abstract}
To understand the dynamics of real-world networks, we investigate a mathematical model of the interplay between the dynamics of random walkers on a weighted network and the link weights driven by a resource carried by the walkers. Our numerical studies reveal that, under suitable conditions, the co-evolving dynamics lead to the emergence of stationary power-law distributions of the resource and link weights, while the resource quantity at each node ceaselessly changes with time. We analyze the network organization as a deterministic dynamical system and find that the system exhibits multistability, with numerous fixed points, limit cycles, and chaotic states. The chaotic behavior of the system leads to the continual changes in the microscopic network dynamics in the absence of any external random noises. We conclude that the intrinsic interplay between the states of the nodes and network reformation constitutes a major factor in the vicissitudes of real-world networks.
\end{abstract}

DOI: 10.1103/PhysRevE.91.012908

PACS number(s): 05.45.-a, 05.65.+b, 89.75.Fb, 89.75.Hc

\section{INTRODUCTION}

Our daily lives are closely related to various biological, engineering and social networks, such as gene regularity networks, neural networks, food webs, communication networks, traffic networks, power grids, and social network services. Extensive studies of these networks have been made in the past decade, revealing the specific statistical structures of the networks and offering insights into the generating mechanisms $[1,2]$. Moreover, many types of dynamical processes on the networks have been studied [3,4]. Today, researchers have begun to focus on the intersections between network structure and dynamics.

Most previous studies have analyzed the dynamical processes that occur on static networks, which comprise fixed structures that do not change with time. In contrast, real-world complex networks are continuously changing with time, in response to alterations in the network states. For example, worldwide traffic and communication networks are constantly developing to meet the evolving needs of society.

This dynamic aspect of complex networks is a growing interest for many disciplines [5,6]. To appreciate how networks will change and to know how to manage them, we need to understand the nature of network dynamics. There is a close interdependency between the reformation of networks and their dynamical processes. For example, consider networks for the transport of people and products between and within cities. As cities develop or decay, traffic networks are frequently reformed to meet current needs, either by the construction of new roads or by the closure of existing roads. Network reformation causes changes in traffic patterns and influences further expansion or contraction in cities. Thus, the reformation of road networks is interdependent with the traffic dynamics. Web browsing traffic is guided by links on the web. Heavy traffic at one site will lead to the creation of additional links to that site. On social network services, such as Facebook and Twitter,

*takaaki.aoki.work@gmail.com people tend to gather around concurrent topics, which induces the drastic formation of new social relationships and initiates further movements in these networks. A similar process occurs in biological systems. In a neural network, the nerve cells in the brain connect to other cells via synapses, whose connections drastically changes in response to neuronal activities. Another example is the gene regulatory network, in which the rates of interactions dynamically change depending on the reaction products of the specific gene regulatory system.

The essence of these changing networks resides in the interplay between two kinds of co-evolving dynamics: specifically, the reformation and dynamical processes of the network. Some interesting issues that arise include the questions of what emerges from this interplay and how the interactions are spontaneously organized. Although this interplay makes the system inherently difficult to analyze, this kind of challenging problem is inherent in physics.

The co-evolving dynamics of adaptive networks has been studied independently in diverse scientific fields, as reviewed by Gross [5]. However, the fundamental properties that are evoked by the interplay have not been well studied, particularly from a systematic perspective. To develop this research area further, some useful toy models are needed that can capture the essences of the dynamic aspect of real-world networks.

In our previous paper [7], we considered a simple model of co-evolving network dynamics, which combines the dynamics of random walkers on a weighted network with the dynamics of the link weights driven by a resource carried by the walkers. The resource quantity defines the state of the node. This model is completely deterministic without any random process. Thus, in this paper, we extensively analyze the emergent network from the viewpoint of the dynamical system. From this detailed analysis, we investigate what type of structured network emerges and what dynamics underlie the network organizations.

This paper proceeds as follows: In Sec. II we describe the model that we use in this paper, which is a little more general than that proposed in Ref. [7]. In Sec. III, we demonstrate that, under feasible conditions, the power-law distributions 
of the resource and link weights emerge spontaneously from the co-evolving dynamics. Under the macroscopic steady-state condition, the amount of resource at individual nodes and their link weights continue to change at the microscopic level. In Sec. IV, we investigate the behavior of the organized scalefree network from the perspective of the dynamical system. We focus on the types and number of attractors and the type of bifurcation that are found in the system. This information reveals the nature of the network that emerges from the coevolving dynamics. Finally, Sec. V summarizes and discuss our findings.

\section{MODEL}

Diffusion is a fundamental process that is related to many physical and social phenomena of real-world networks, such as traffics, transports, human mobility, information disseminations, and epidemic spreading [3,4,8]. Therefore, we consider a diffusion process on a weighted network as a paradigm for dynamical processes on networks. The state of each node at time $t$ is represented by the current amount of the diffusive resource at the node, $x_{i}(t)$. In real-life situations, the resource may be molecules, cells, people, money, data packets, and so on. The network topology with $N$ nodes is given by an adjacency matrix $a_{i j}$. The link weight from the $j$ th to $i$ th node for each existing link is denoted by $w_{i j}$. We assume that the resource diffuses over a weighted network carried by many random walkers. Then, the diffusion process is described by

$$
\frac{d x_{i}(t)}{d t}=F\left(x_{i}(t)\right)+D \sum_{j \in \mathcal{N}_{i}}\left[T_{i j}(t) x_{j}(t)-T_{j i}(t) x_{i}(t)\right],
$$

where $D$ is a diffusion scale parameter that controls the strength of diffusion over the whole system. The diffusion matrix $T_{i j}(t)$ is given by $w_{i j}(t) / s_{j}(t)$ and $s_{j}(t)\left[=\sum_{i} w_{i j}(t)\right]$ is the strength of the node $j . \mathcal{N}_{i}$ is the set of nodes connected to the $i$ th node. The second and third terms are the inward and outward currents of the resource, respectively. The first term $F(x)$ describes a reaction process at the node, which represents the intrinsic dynamics of the resource except for the diffusion process. We assume a simple dissipation process with an equilibrium state, given by

$$
F(x)=-\kappa(x-1),
$$

where $\kappa$ is a decay constant. The total amount of resource over the network, $\sum_{i} x_{i}(t)$, always converges to $N$, according to the equation, $\frac{d \sum_{i} x_{i}(t)}{d t}=-\kappa\left[\sum_{i} x_{i}(t)-N\right]$. To maintain the total amount of resource, the initial amounts of the resource at the nodes are constrained to satisfy the condition $\sum_{i} x_{i}(0)=N$.

A significant feature of the network is that the diffusion matrix $T_{i j}(t)$ changes with time depending on the amounts of resource. For the sake of simplicity, we assume that the topology of the network $a_{i j}$ is constant and that the link weights $w_{i j}(t)$ for existing links $\left(a_{i j}=1\right)$ are time dependent. Thus, we consider the dynamics of the link weights (i.e., interaction strength) to be a function of the resource.

The amount of resource represents the centrality of the node. For example, a link to a resource-rich (or resourcepoor) node tends to be strong (or weak). The interaction strength, $w_{i j}(t)$, will be proportional to $x_{i}(t)$ and $x_{j}(t)$. It

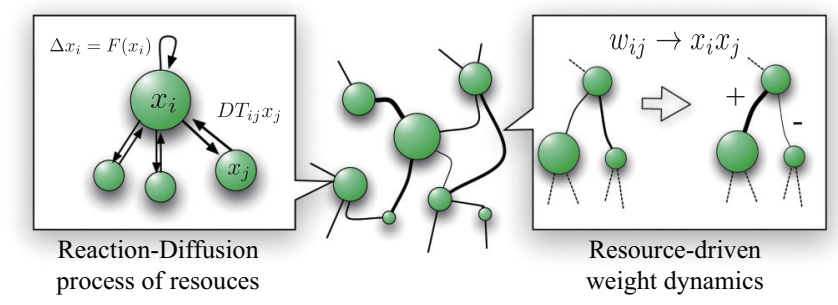

FIG. 1. (Color online) Schematic illustration of co-evolving network dynamics, combining the dynamics of the resource carried by random walkers and the resource-driven dynamics of the link weights.

is straightforward to introduce a generalized form of the nonlinear dependency on $x_{i}(t)$ and $x_{j}(t)$. Then, we introduce a simple relaxation process, similar to the law of mass action, in the form

$$
\frac{d w_{i j}(t)}{d t}=\epsilon\left[x_{i}(t) x_{j}(t)-w_{i j}(t)\right],
$$

where $\epsilon$ controls the relaxation time scale of the weights. Equation (2) indicates that the richer the resource-containing node is, the stronger the links will be that connect to the node. If a link weight approaches zero due to a poor resource on the endpoint node, then the link is effectively removed. In this sense, the dynamical process determines the effective topology of the network.

Finally, by combining Eqs. (1) and (2), we obtain the equations for the model (Fig. 1):

$$
\begin{gathered}
\frac{d x_{i}(t)}{d t}=\kappa-(D+\kappa) x_{i}(t)+D \sum_{j \in \mathcal{N}_{i}} \frac{w_{i j}(t)}{s_{j}(t)} x_{j}(t), \\
\frac{d w_{i j}(t)}{d t}=\epsilon\left[x_{i}(t) x_{j}(t)-w_{i j}(t)\right] .
\end{gathered}
$$

We assume that the underlying network topology is undirected, and that the weights of the links are symmetric. In the numerical simulations, we use the following map equations by solving the ordinary differential equation system by the Euler method:

$$
\begin{gathered}
x_{i}(t+1)=\kappa+(1-\kappa-D) x_{i}(t)+D \sum_{j \in \mathcal{N}_{i}} \frac{w_{i j}(t)}{s_{j}(t)} x_{j}(t), \\
w_{i j}(t+1)=(1-\epsilon) w_{i j}(t)+\epsilon x_{i}(t) x_{j}(t) .
\end{gathered}
$$

The time step width $h$ can be absorbed into the parameters that characterizes the model, $D h \rightarrow D, \kappa h \rightarrow \kappa$, and $\epsilon h \rightarrow \epsilon$. Each of these dynamics is trivial by itself. If the network is static (i.e., the weights are time-independent), then Eq. (5) of the resource has a unique stable fixed point, according to the Perron-Frobenius theorem. On the other hand, if the resource distribution is static, then the weights of the links are regulated by a negative feedback with decay time constant $\epsilon^{-1}$, adjusted to the resource quantity. However, the combined system exhibits nontrivial behaviors (as shown later in Sec. III), revealing the complex properties of changing networks. Thus, we focus on the parameters for cases in which the time scales of both dynamics are comparable. 

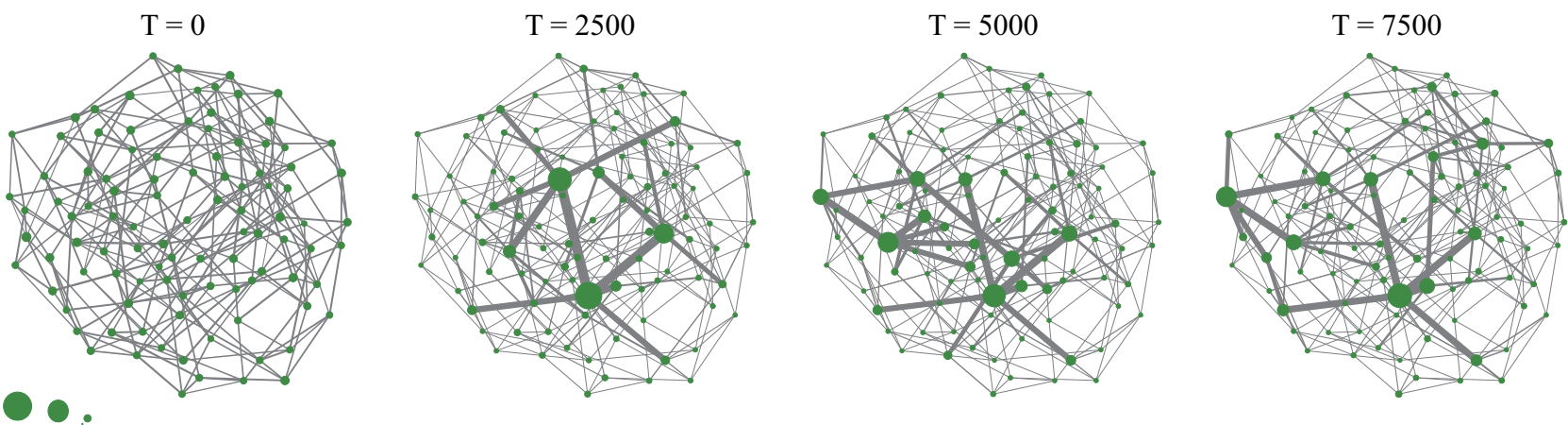

20101
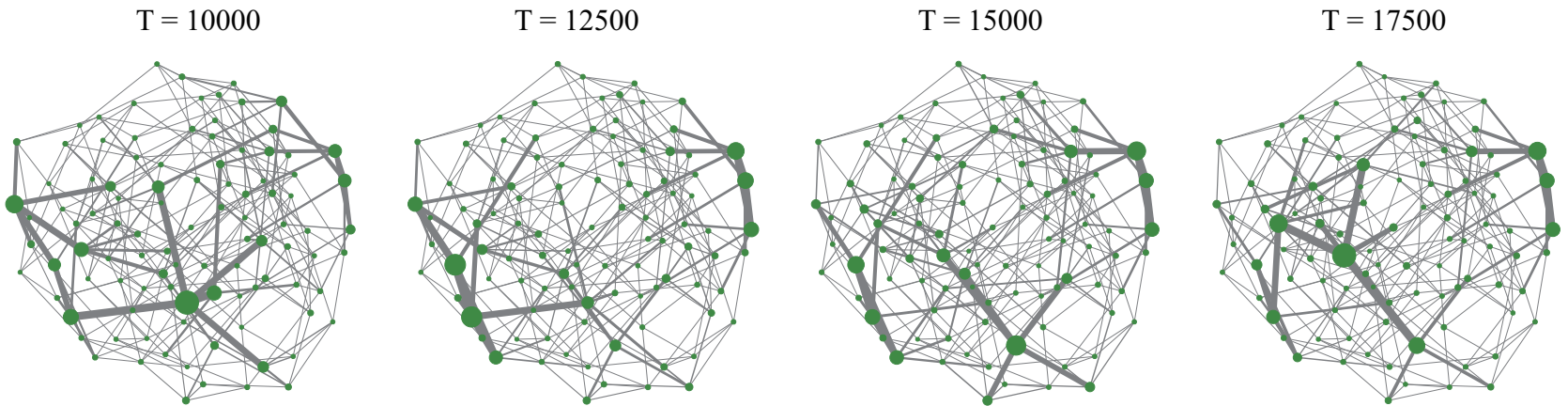

FIG. 2. (Color online) Typical behavior of the co-evolving dynamics. At each time, the resource is concentrated into a few nodes, which strengthens the link weights around them. Resource quantities at the nodes and the link weights between them continue to change forever at the microscopic level, whereas they converge to heterogeneous distributions at the macroscopic level. The parameters are as follows: $\kappa=0.05$, $D=0.34$, and $\epsilon=0.01$.

\section{POWER-LAW DISTRIBUTIONS OF THE RESOURCE AND THE LINK WEIGHTS IN THE EQUILIBRIUM STATE}

Figure 2 displays the typical behavior of the co-evolving dynamics described by Eqs. (5) and (6). In this figure, the size of a circle represents the amount of resource at a node. Initial values are generated by a normal distribution with mean $\mu=1$ and standard deviation $\sigma=0.1$. The initial resource and weight distribution are almost homogeneous. The underlying fixed topology is given by a regular random graph, with size $N=100$ and degree $k=5$. Other parameter values are as follows: $\epsilon=0.01, \kappa=0.05$, and $D=0.34$. From the figure, it can be observed that the resource initially becomes concentrated in some nodes, which become hubs. During the first interval, the resource accumulates at a few nodes near the center of the graph, and the weights of the connections between these resource-rich nodes are strengthened. As other nodes develop into hubs, drastic changes occur in the resource distribution. Nodes that were previously hubs lose their hub status, and the links connecting them are weakened. After these initial changes, the network repeatedly evolves in a similar manner (see movie S1 in the Supplemental Material for reference [64]).

We next focus on the statistical properties of a large organized network ( $N=16384)$. The underlying topology is given by an Erdös-Rényi random graph with $\langle k\rangle=10$. Figure 3 shows the resource distribution and link weights after a transient period, which take the form of a power (a)

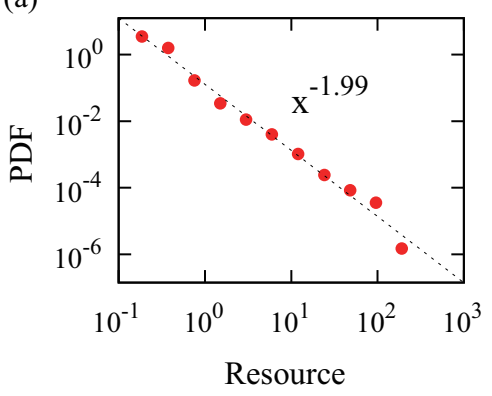

(b)

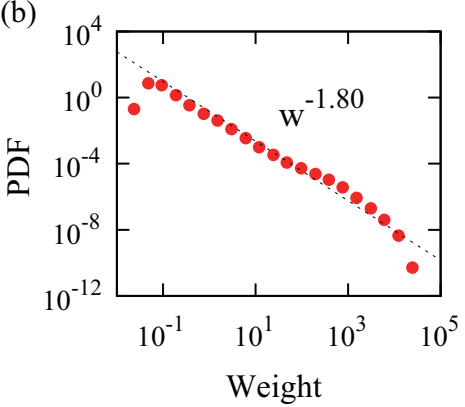

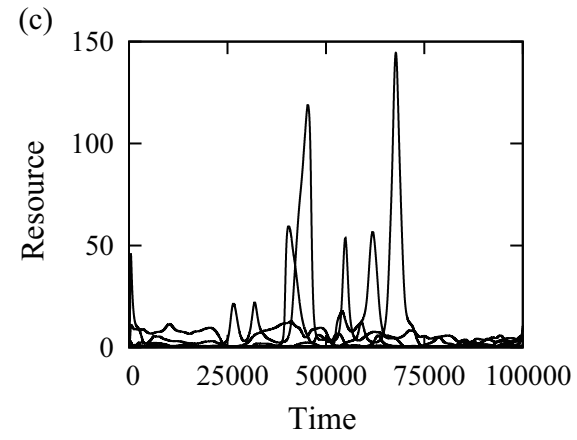

FIG. 3. (Color online) (a) Distribution of the resource quantity $x_{i}$ in the equilibrium state for a large network. Parameters are the same as in Fig. 2, except that the underlying network is given by an Erdös-Rényi random graph $(N=16384,\langle k\rangle=10)$. (b) Weight distribution. (c) Time developments of the resource quantities for several selected nodes. 
(a)

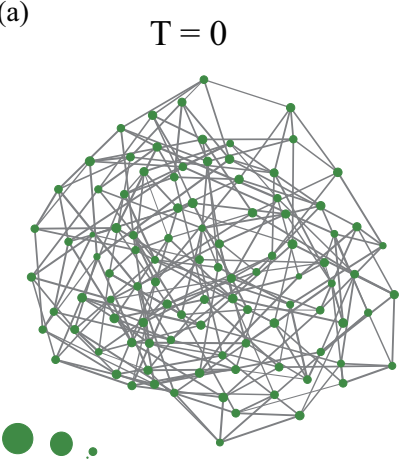

20101

(b)

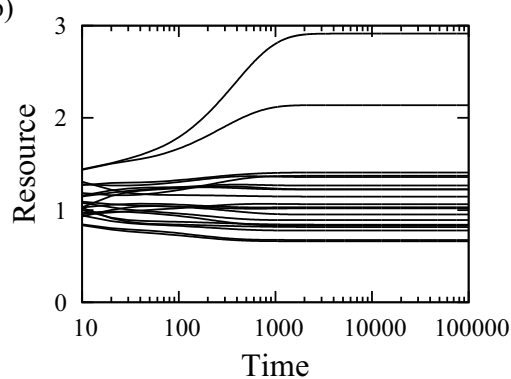

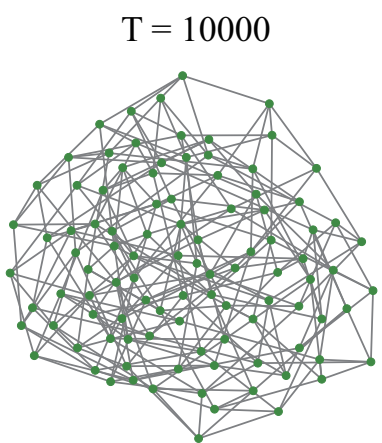

(c)

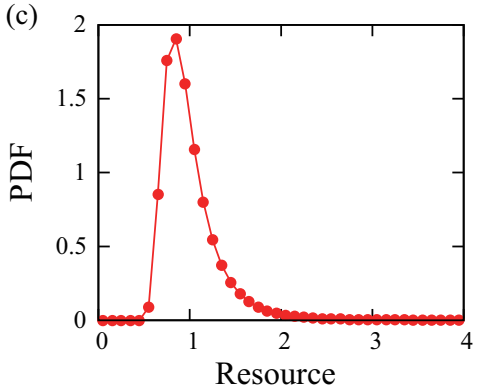

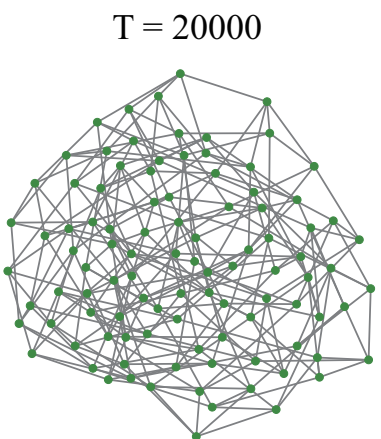

(d)

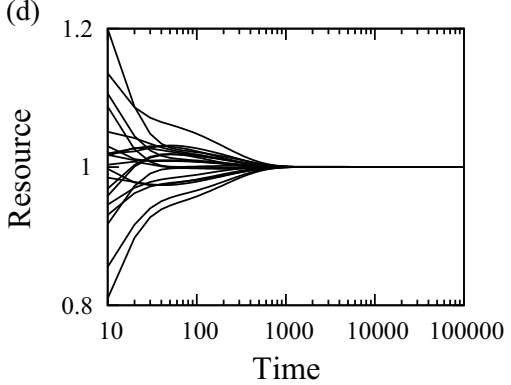

FIG. 4. (Color online) Another model parameter with lower $D$ leads to the organization of the homogeneous network. Parameters are the same as in Figs. 2 and 3, except that $D=0.05$. (a) Typical behavior of a small network. Changes of the resource quantities at nodes are difficult to see in the scale of the graphs. (b) Time development of the resource at several sampled nodes in a large network (Erdös-Rényi random graph with $N=16384,\langle k\rangle=10$ ). Values converge to different constants. (c) Resulting resource distribution in the organized network. (d) Same plot as in (b), except that the underlying network is given by regular random graphs with $k=10$.

law. In this case, the exponent of the resource distribution is approximately -2 (for a cumulative distribution, the exponent is -1). This result is consistent with Zipf's law [9], an empirical law that can be observed in the many resource distributions in real-world networks [10-14]. The weight and resource distribution have different exponents.

Although the resource quantities converge to a stationary power-law distribution at the macroscopic level, they continue to change with time at the microscopic level. Figure 3(c) shows the temporal evolution of the resource quantities at several nodes, where the quantities show irregular changes with time.

Using other parameters with a lower diffusion constant $D$, the system converges to an alternative network type, in which the microscopic resource dynamics are frozen, and the resource distribution does not adhere to a power law (Fig. 4). The resource variation between nodes originates from variation in the underlying network. In fact, on the regular random graph, the resource quantities converge to be the same value at all nodes. Thus, this equilibrium state is intrinsically homogeneous.

The emergence of these two types of network organizations can be controlled by the model parameters. The system particularly depends on $\kappa$ and $D$, which determine two types of resource flows:

$$
\begin{aligned}
& x_{i}(t+1)-x_{i}(t) \\
& \quad=\kappa-\kappa x_{i}(t)+D \sum_{j \in \mathcal{N}_{i}}\left[\frac{w_{i j}(t)}{s_{j}(t)} x_{j}(t)-\frac{w_{i j}(t)}{s_{i}(t)} x_{i}(t)\right] .
\end{aligned}
$$

The diffusion constant $D$ controls the resource flow via the weighted network, based on the local interaction between nodes. The decay constant $\kappa$ determines the global redistribution of the resource. At each time step, each node pays a percentage of the resource, $\kappa x_{i}(t)$. The summation of these percentages $\left[=\kappa \sum x_{i}(t)=\kappa N\right]$ is equally redistributed among the nodes, by the term $+\kappa$, and the balance between the two distinct flows determines the resource distribution. Figure 5 shows the exponent of the probability density distributions of the resource and link weights on $(D, \kappa)$ space, where $\epsilon=0.01$. The underlying network is given by a Erdös-Rényi random graph $(N=16384,\langle k\rangle=10)$. In the colored area, the distribution takes the form of a power law, whose exponents are indicated by the color coding on the right. The distributions are fitted by the method proposed in Ref. [15]. The dominant local flow is controlled by diffusion constant $D$, and the resource distribution has significant inequality among the nodes. In the white area, the dominant distribution is global redistribution by $\kappa$. This redistribution tends to homogenize the resource distribution, which does not take the form of a power law [inset in Fig. 5(a)]. Thus, the model exhibits two distinct types of network organizations, without an intermediate state between these regions. This finding implies that the transition between homogeneous and heterogeneous resource distributions occurs at the boundary between those areas. We will analyze this transition in the next section.

To evaluate the unsteady microscopic dynamics observed at the equilibrium state, we consider the rate of changes of the 

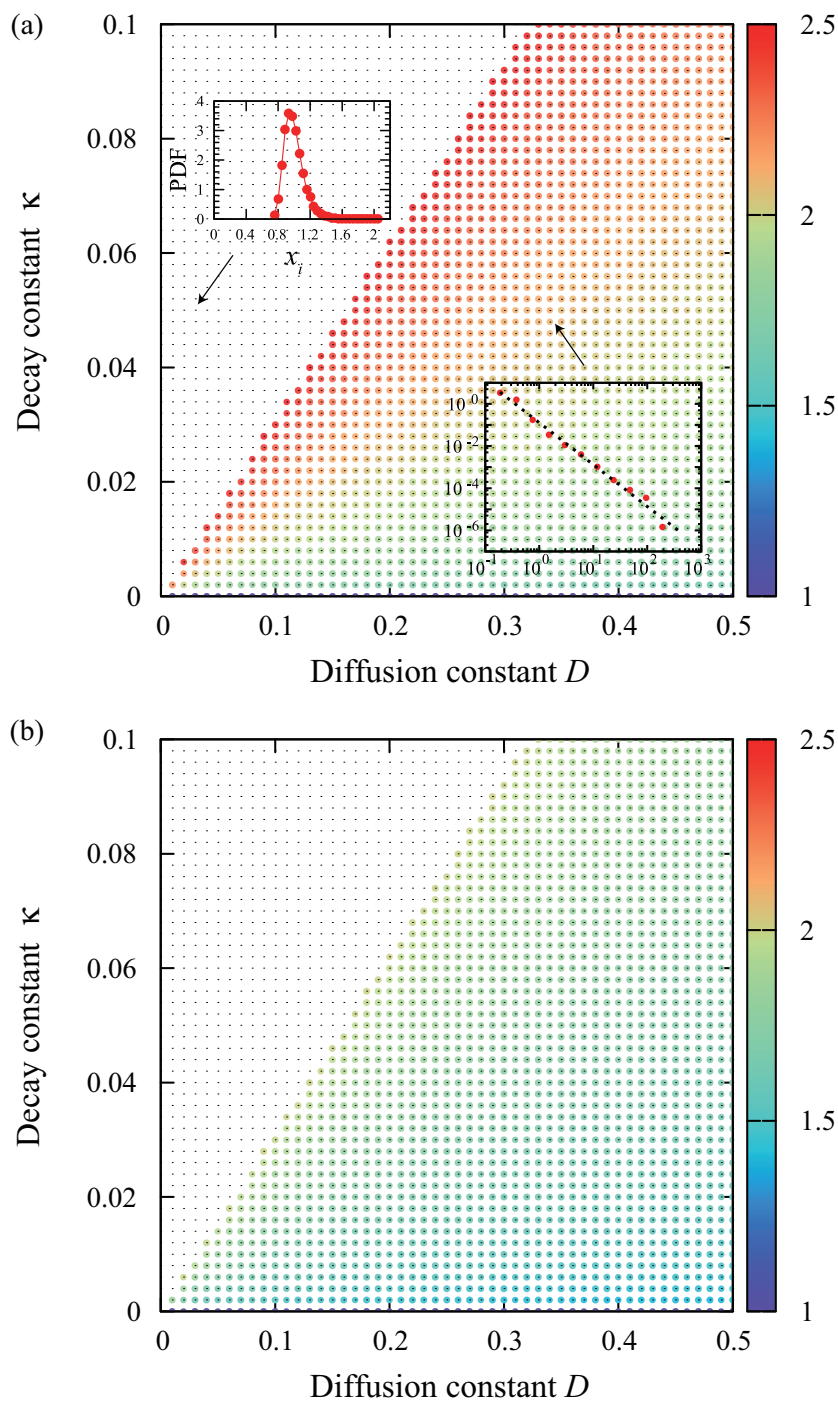

FIG. 5. (Color) (a) Exponents of the power-law distributions of the resource, $\gamma_{x}$, in $(D, \kappa)$ space. In the colored area, the distribution takes the form of a power law, whose exponents are indicated with the color coding on the right. In the white area, the distribution does not take the power law form. (b) Exponents of the power-law distributions of the link weights, $\gamma_{w}$, in $(D, \kappa)$ space.

resource given by

$$
V(t)=\frac{1}{N} \sum_{i}\left|x_{i}(t)-x_{i}(t-1)\right|
$$

and evaluate the time-averaged rate $\langle V\rangle$ at the equilibrium state. As shown in Fig. 6 , the $(D, \kappa)$ space is clearly divided into an unsteady area (nonzero $\langle V\rangle$ ) and a frozen area (zero $\langle V\rangle$ ), consistent with the regions in Fig. 5. This result implies that the nearly homogeneous distribution of the resource is frozen at the microscopic level and that the heterogeneous powerlaw distribution of the resource has unsteady microscopic dynamics.

Exponents $\gamma_{x}$ and $\gamma_{w}$ of the power-law distribution of the resource and the link weights depend on the model parameters (Fig. 5). In the parameter region of the power-law state (colored region), a relationship exists between these exponents (Fig. 7).

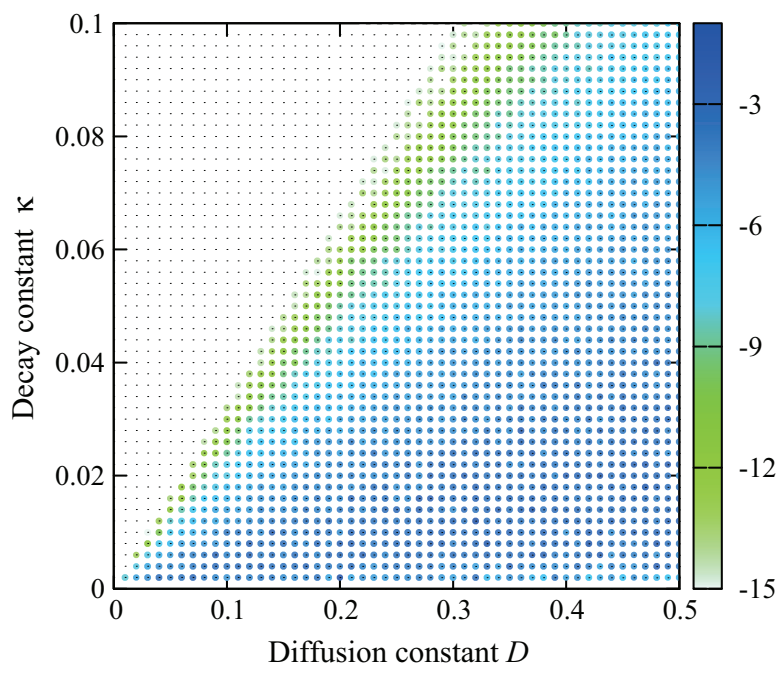

FIG. 6. (Color) Rate of change of the resource, measured by $\log \langle V\rangle$ in Eq. (7), plotted in $(D, \kappa)$ space.

The red and blue points show the numerical results in the case of an Erdös-Rényi random graph and a regular random graph, respectively. The two lines indicate the theoretical prediction under the extreme cases that the resource quantities at adjacent nodes are uncorrelated and are equal, respectively. These assumptions yield the following formulas for the exponents $\gamma_{x}, \gamma_{w}$ (see the Appendix):

$$
\begin{gathered}
\gamma_{w}=\gamma_{x} \quad(\text { Uncorrelated }), \\
\gamma_{w}=\frac{\gamma_{x}+1}{2} \quad \text { (Equal). }
\end{gathered}
$$

Figure 7 indicates that the numerical data do not match with the theoretical predictions in these two extreme cases. The amounts of resource are not equal at the adjacent nodes, but there is a correlation between the amounts.

The parameter $\epsilon$ controls the relaxation time scale of the weights. Figure 8 plots the $\epsilon$ dependence of the exponent of the

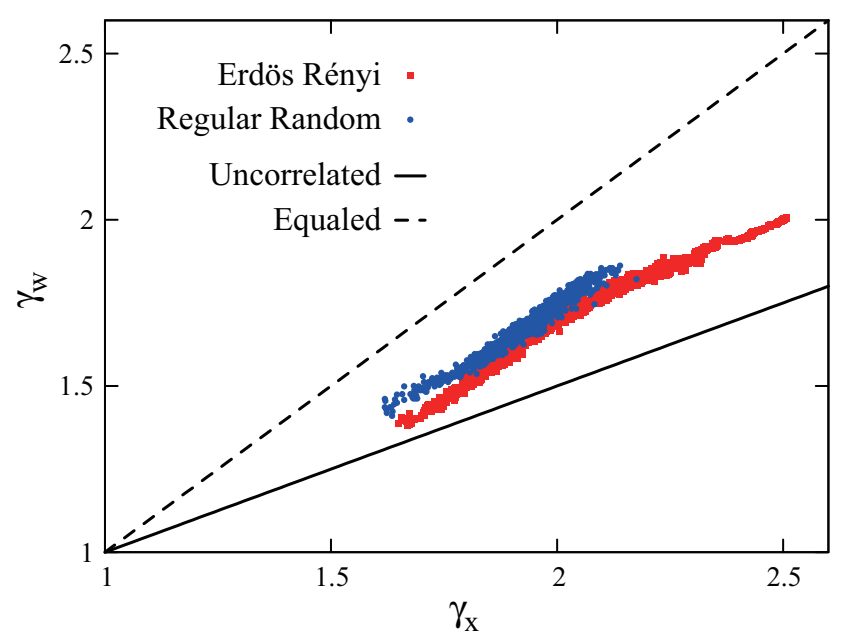

FIG. 7. (Color online) Relationship between the exponents of resource $\gamma_{x}$ and link weights $\gamma_{w}$. Points indicate the numerical results. Lines show the theoretical predictions under two extreme conditions. 


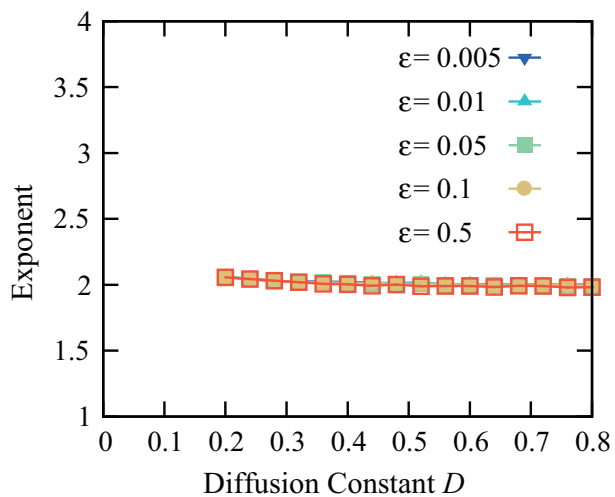

FIG. 8. (Color online) $\epsilon$ dependence of the organized network. Graph plots the exponent of the power-law resource distribution as a function of $D$. Parameters are the same as in Fig. 5, except that $\kappa=0.05$.

power-law distribution of the resource at the equilibrium state. This figure suggests that there is no significant dependence on $\epsilon$.

The underlying network topology is an implicit parameter of the model. Theoretical studies often consider the all-to-all coupling and the mean field approximation, which are useful techniques for analyzing network dynamics in general $[3,16]$. Although the above results are robustly confirmed in several underlying network topologies, surprisingly, the complete

(a)

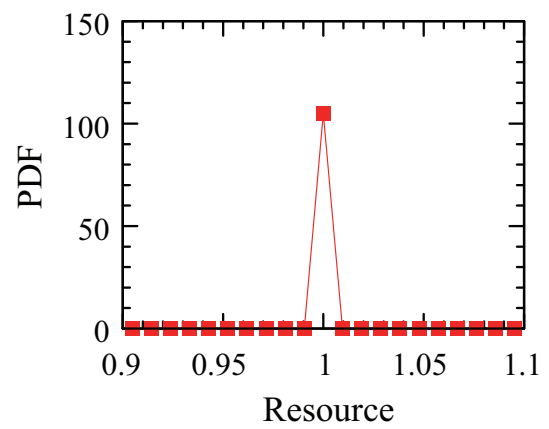

(c)
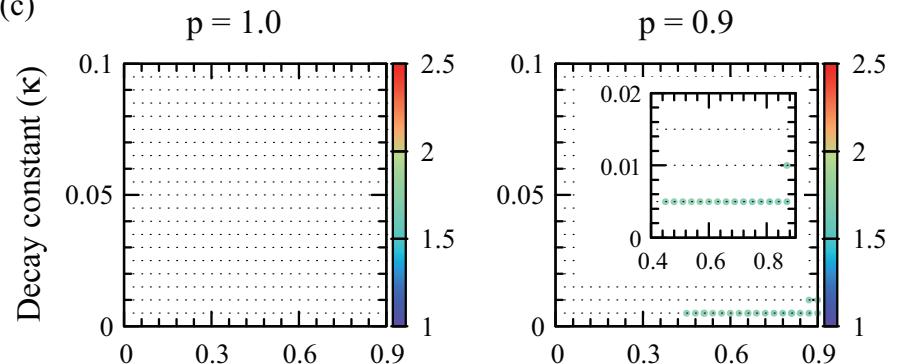

graph (i.e., with all of nodes connected to other nodes) exhibits an exceptional and qualitatively unique behavior. In the entire range of possible parameters, the system always converges to the completely homogenous state (i.e., $x_{i}=w_{i j}=1$ ). This anomalous behavior seems to be structurally unstable, because the completely homogeneous solution disappears from the underlying network when the complete graph is slightly modified (a few links are moved). We numerically confirm this unstable behavior in the case of $\kappa=0$ (with $\epsilon=0.5, D=1.0$ ). On the perfectly complete graph, the resource amounts at all nodes converge to the same value [Fig. 9(a)]. However, on an almost complete graph $(0.1 \%$ of links are removed), a heterogeneous resource distribution emerges [Fig. 9(b)] that asymptotically converges to a power-law distribution. This distribution has different properties from the power-law distribution in the case of $\kappa \neq 0$. The resource-poorest node, $\min _{i} x_{i}(t)$, converges to zero resource, while the resource-richest node almost keeps its value. Thus, the resource distribution broadens as time elapses. For more details of the numerical and analytical results in this case, see Ref. [7]. In the general case of $\kappa \neq 0$, we numerically obtain the phase diagram on $(D, \kappa)$ space for various values of the connection probability $p$ in an Erdös-Rényi random graph [Fig. 9(c)]. On the complete graph, there is no parameter region of the heterogeneous resource distribution. As more links are removed from the complete graph, the region becomes broader. Based on these numerical results, we conjecture that the underlying networks must be sparse in order for a stationary power-law distribution to emerge, and that the (b)

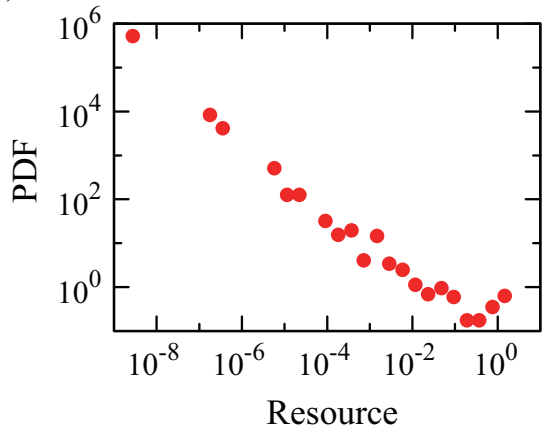

Diffusion constant $D$

FIG. 9. (Color) The model exhibits a structurally unstable behavior that only appears if the underlying network is given by the complete graph. In the entire range of the possible parameters, the system always converges to the completely homogeneous state, i.e., $x_{i}=w_{i j}=1$. (a) On the perfectly complete graph, the resource quantities at all nodes converge to the same value. (b) Slight modification of the complete graph $(0.1 \%$ of links are removed) destroys the resource configuration in (a), and a heterogeneous resource distribution emerges. (c) Exponents $\gamma_{x}$ of the power-law distributions of the resource in $(D, \kappa)$ space for various connection probabilities $p$ in an Erdös-Rényi random graph. Parameters are the same as in Fig. 5, except for $N=1024$. 
mean field approximation is not suitable for analysis of this model.

\section{ANALYSIS OF THE MICROSCOPIC DYNAMICS OF THE ORGANIZED NETWORK}

In Sec. III we have numerically revealed that the model exhibits two distinct types of network organizations depending on the parameters: a static resource configuration with a nearly homogeneous distribution and a configuration with continuous microscopic changes and an asymptotic power-law distribution. Next, we investigate the microscopic structure of the organized network in terms of the resource distribution, by analyzing the equilibrium state from the perspective of a dynamical system.

First, we derive a fixed point for Eqs. (5) and (6). The condition for a fixed point $\left(x_{i}^{*}, w_{i j}^{*}\right)$ is given by

$$
\begin{aligned}
x_{i}^{*} & =\frac{1}{\frac{D}{\kappa}\left(1-\sum_{j} \frac{x_{j}^{*} a_{i j}}{\sum_{k} x_{k}^{*} a_{j k}}\right)+1}, \\
w_{i j}^{*} & =x_{i}^{*} x_{j}^{*} .
\end{aligned}
$$

This condition seems to be complicated, but for a constantdegree network (e.g., a regular random graph, ring, lattice, or complete graph), we can find a solution to satisfy the condition. Given that the degree, $\sum_{j} a_{i j}$, is constant for all $i$, there is a completely homogeneous solution given by $x_{i}^{*}=w_{i j}^{*}=1$ for all nodes.

To identify the transition point from a homogeneous to a heterogeneous distribution of the resource in $(D, \kappa)$ space, we focus on the case that the underlying topology is given by a regular random graph and analyze the homogeneous solution. The linear stability of the homogeneous solution is numerically evaluated in a network of 100 nodes with $k=5$ and $\epsilon=0.1$ (Fig. 10). In Fig. 10(a), the solution is stable in the blue region and unstable in the red region. Figure 10(b) shows the results obtained from the full simulation of the model equations. In the blue region, the resource distribution converges to a homogeneous distribution; in the red region, there is an unequal distribution of resource at the nodes. The results of the linear stability analysis are consistent with those of the (a)

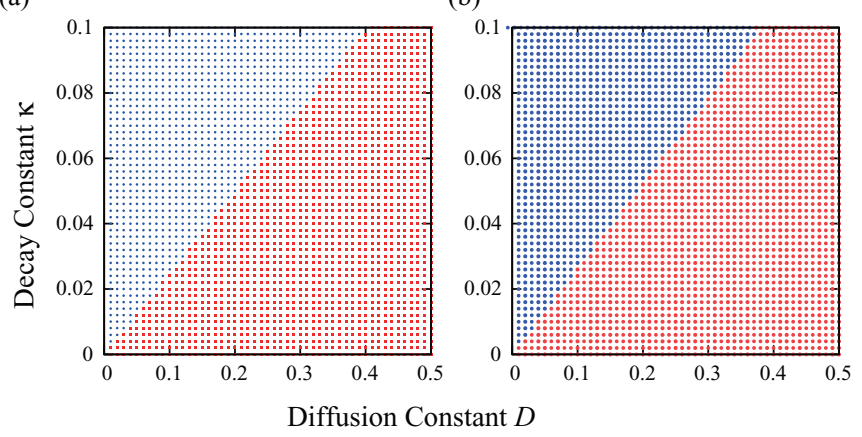

FIG. 10. (Color) (a) Liner stability of the homogeneous solution, i.e., $x_{i}=w_{i j}=1$ for all nodes, in the case of a regular random graph. Solution is stable in the blue region and unstable in the red region. (b) Stable (blue) and unstable (red) regions of the homogenous solution obtained from the full simulation of the model equations. full simulation. Thus, the destabilization of the homogeneous solution determines the transition from a homogeneous to a heterogeneous resource distribution.

When destabilization occurs, the system exhibits unsteady microscopic dynamics with a stationary power-law distribution, as shown in the previous section. The dynamical system of our model is completely deterministic. Therefore, it is pertinent to ask what the underlying dynamics behind such unsteady behaviors may be. We propose three candidates to explain the numerical results. The first candidate is a very long-term relaxation toward a fixed point. In this case, the microscopic dynamical behavior is transient, although the randomness of the connections makes the relaxation time very long. The second candidate is a periodic state, which looks irregular because of its long periodicity. The third candidate is a chaotic state, in which the system intrinsically and continuously exhibits irregular behavior. To identify which of the above candidates is responsible for the observed behavior, we numerically evaluate two measures: the total displacement rate and the maximum Lyapunov exponent. We consider small networks with 512 nodes $(k=5)$, although the degree of freedom in the system is somewhat large (1792 dimensions). The total displacement rate is given by $|\mathbf{z}(t+1)-\mathbf{z}(t)|$, where $\mathbf{z}(t)=\left\{x_{i}(t), w_{i j}(t)\right\}$. If this rate converges to zero after a long time, then the system is settled in a fixed state. Otherwise, if the maximum Lyapunov exponent is positive, then the state is chaotic. In the remaining case, it seems to be periodic. Figure 11 shows the typical behaviors of the above three dynamics. The initial conditions determine which dynamics emerge in the system. In Fig. 11(a), the system finally settles on a fixed point after some transient period. The total displacement rate converges to zero, and the maximum Lyapunov exponent is negative. This is another fixed point satisfying the condition (10), which is completely different from the homogenous solution. In Fig. 11(b), the system remains in a periodic state after a transient period. The displacement rate does not converge to zero, but oscillates around some finite value with a small amplitude. The maximum Lyapunov exponent is nearly zero. In the middle graph, the time developments of the resource quantities exhibit periodic motions at some sampled nodes. In Fig. 11(c), the system is in a chaotic state. The Lyapunov exponent is positive, and each resource quantity exhibits irregular changes at the microscopic level. Such differences in microscopic behavior originate from the multistability of the system. Even if the network has the same topology $a_{i j}$, the three types of microscopic behavior are still observed depending on the initial conditions of the resource and weights. Despite these distinct microscopic behaviors, we find no significant statistical difference among the resource distributions. These distributions take a form of power law, in which the exponents are almost the same in all three cases. In other words, these distinct states have a similar, heterogeneous distribution of the resource at the macroscopic level, whereas their microscopic behaviors are definitely different. In the fixed states, the node ranking in respect of the resource amount is frozen. On the other hand, in the periodic and chaotic states, the ranking always changes with time, keeping the characteristics of the heterogeneous resource distribution. This result might suggest that the motion of the system in the phase space is constrained on a certain 
(a)

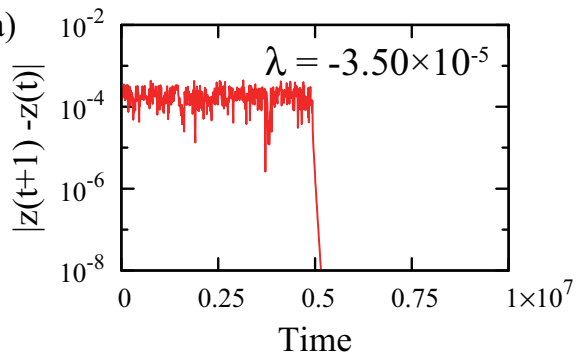

(b)

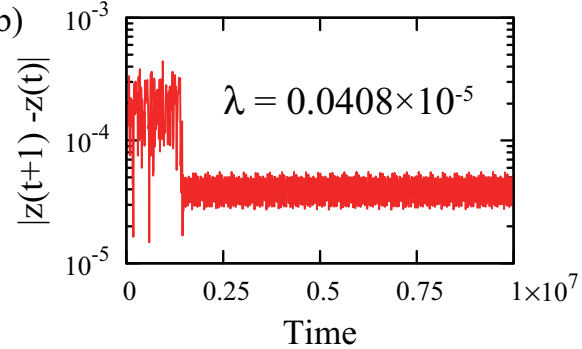

(c)

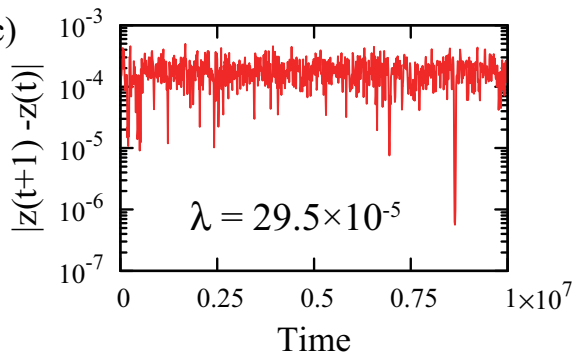

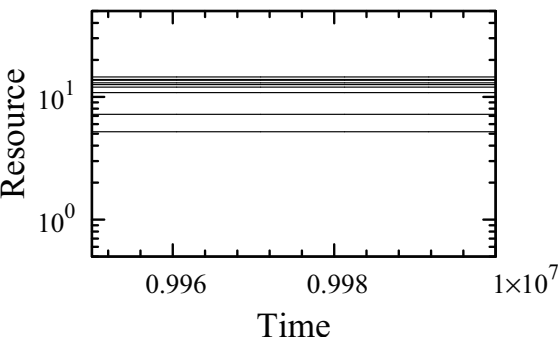
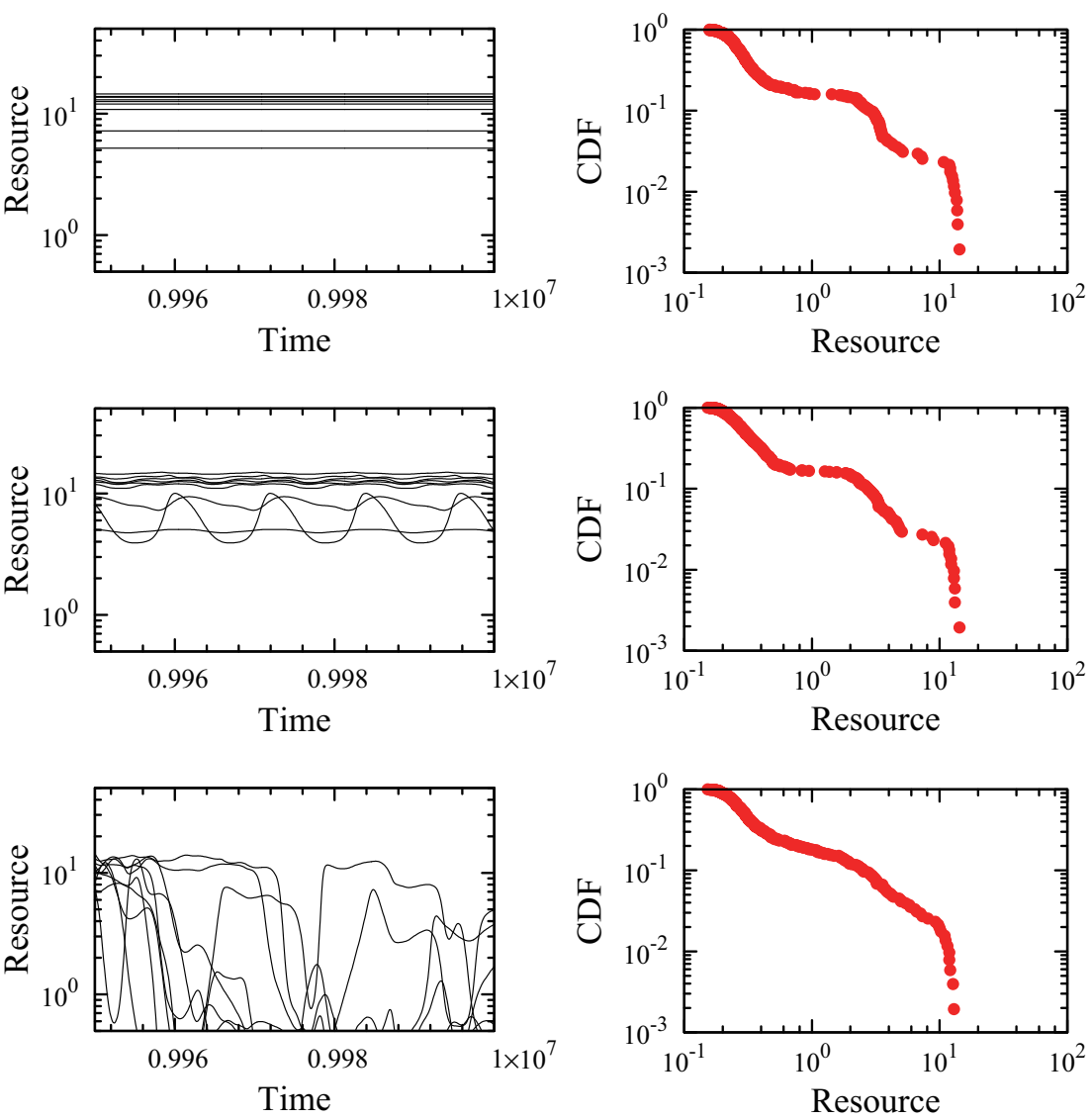

FIG. 11. (Color online) Three types of microscopic behaviors seen in the case of a small network $(N=512, k=5, \kappa=0.05, D=0.4$, and $\epsilon=0.01$ ). (a) Fixed point with a long relaxation time. (b) Limit-cycle oscillation with a long periodic time. (c) Chaotic state. Graphs in the left column depict the total displacement rate given by $|\mathbf{z}(t+1)-\mathbf{z}(t)|$, where $\mathbf{z}(t)=\left\{x_{i}(t), w_{i j}(t)\right\}$, where $\lambda$ is the maximum Lyapunov exponent. Graph in the middle column shows the time development of the resource at several nodes. Graph in the right column indicates the cumulative resource distribution.

hyperplane satisfying the heterogeneous resource distribution in the macroscopic level. There are many fixed points, limit cycles, and chaotic attractors on this hyperplane. Depending on the initial state, the system converges either of them.

Given that the initial condition is randomly generated by a normal distribution $(\mu=1.0, \sigma=0.25)$, we investigate the percentages of the three states. Numerous distinct fixed points and limit cycles are included within the percentages of the fixed and periodic states. Figure 12 shows the results of numerical simulations conducted until time step $t=T_{s}$ and the maximum Lyapunov exponent calculated using only the last $T_{0}\left(=2 \times 10^{6}\right)$ steps to determine the percentages of the three states. In other words, we consider the time $T_{s}-T_{0}$ to be the transient period for convergence to a fixed or periodic states. Figure 12(a) shows the dependence of the percentages of the three states on $T_{s}$. As $T_{s}$ increases, the percentage of the chaotic state decreases, and the percentages of the fixed and periodic states gradually increase. This result occurs because the system chaotically evolves with time in the first steps and occasionally is trapped at fixed points or limit cycles. Interestingly, the profile of the percentages is almost stationary for a large $T_{s}$, and a certain percentage of the chaotic state exists. However, this scenario does not rule out the possibility that the system will go to a nonchaotic state after a very long-term relaxation. Figure 12(b) shows the dependence on network size $N$ for
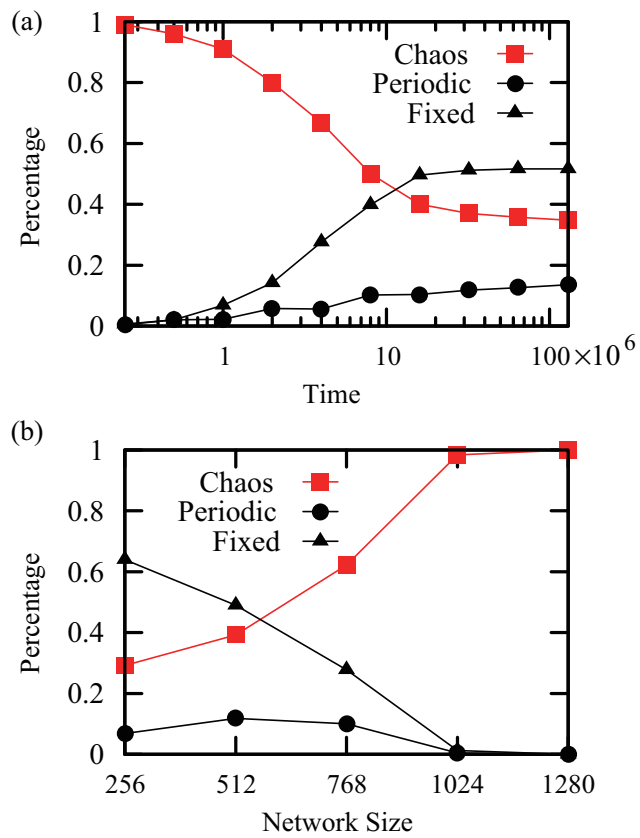

FIG. 12. (Color online) Percentages of the fixed, periodic, and chaotic states. (a) Dependence on $T_{s}$ for $N=512$. (b) Dependence on the network size $N$ for $T_{s}=1.28 \times 10^{8}$. 
(a)

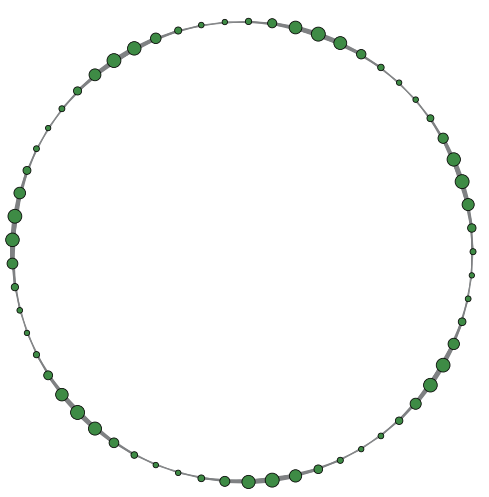

(b)

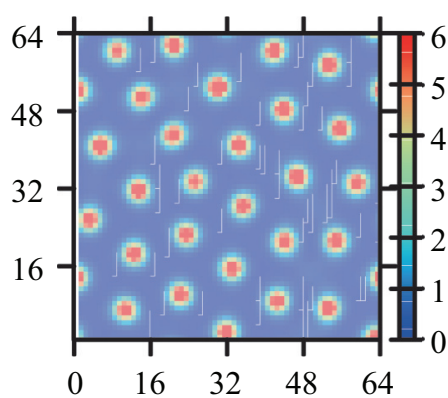

FIG. 13. (Color) Pattern formation on the regular lattices. (a) The destabilization of the homogeneous solution under a regular, ring topology induces a Turing-like wave of the resource distribution. (b) A static spot pattern is observed on a two-dimensional lattice. Other parameters are the same as in Fig. 3.

the largest $T_{s}\left(=1.28 \times 10^{8}\right)$. The percentage of the chaotic state reaches $100 \%$ as the network size increases, which indicates that the relaxation time to a fixed or periodic state increases as the network size grows. This finding implies that the microscopic behavior of a large-scale network is always irregular.

The model shows some specific behaviors if the underlying network is given by regular lattices, in which the homogeneous solution also exists. On a one-dimensional ring topology, the destabilization of the homogeneous solution induces a Turing-like pattern at steady state [Fig. 13(a)]. A static spot pattern is similar observed on a two-dimensional lattice [Fig. 13(b)]. These Turing-like patterns are destroyed by doping the regularity of the underlying network (e.g., by randomly rewiring several links or setting nonuniform diffusion parameters).

Finally, we summarize the microscopic behavior of the model in the case of a regular random graph. There is a homogeneous state where $x_{i}=w_{i j}=1$ for all nodes. The stability of this solution determines the transition between the homogeneous and heterogeneous networks. In the unstable region, there are several types of distinct behaviors (i.e., fixed point, periodic, and chaotic states). However, despite these different microscopic behaviors, the stationary power-law distribution of the resource is almost qualitatively the same. The system has numerous distinct fixed points and periodic states, and the relaxation times toward them are extremely long in a large-scale network. Consequently, the system always behaves chaotically and is occasionally trapped at a fixed point or periodic state after a long transient period.

\section{DISCUSSION}

In this study, we have introduced a simple model of the coevolving dynamics of real-world networks, which combines the dynamics of random walkers and link weights, driven by the amount of resource carried by the walkers. Numerical studies of this model have revealed that, under suitable conditions, the co-evolving dynamics organize stationary power-law distributions of the resource and link weights, in which the resource quantity at each node continues to change with time at the microscopic level. The linear stability of the homogeneous solution for a regular random graph indicates the boundary of the parameter region, determining whether a power-law distribution can emerge for the resource. Furthermore, an analysis of the maximum Lyapunov exponent on the continuous microscopic dynamics has revealed that this system exhibits multistability, including numerous fixed points, limit cycles, and chaotic states.

In the asymptotic state, the resource quantity at each node is essentially equivalent to the PageRank centrality of the node. Under the condition $\sum_{i} x_{i}=N$ (as in our simulations), Eq. (5) can be rewritten by

$$
\Delta \boldsymbol{x}(t)=[\kappa \boldsymbol{G}+D \boldsymbol{T}(t)+(1-\kappa-D) \boldsymbol{I}] \boldsymbol{x}(t),
$$

where $\quad \boldsymbol{x}(t)=\left\{x_{1}(t), x_{2}(t), \ldots, x_{N}(t)\right\}, G_{i j}=1 / N, T_{i j}(t)=$ $w_{i j}(t) / s_{j}(t)$ and $I$ is the identity matrix. If $\kappa+D=1$, then this equation gives the well-known map iteration that is used to evaluate the PageRank. The parameter $\kappa$ corresponds to the dumping factor, which controls the global teleportation of walkers over the entire network. In this sense, the proposed model represents the co-evolving dynamics between the node's centrality measured by the PageRank and the link weights. This finding will evoke future studies of the co-evolving dynamics using another centrality of the node, such as closeness or between centrality.

Historically, the concept of co-evolving network dynamics has primarily been discussed within the neuroscience field [17]. In neural networks, synapses connect the neurons. Their efficiency, often referred to as the synaptic weight, drastically changes depending on neuronal activity. As a fundamental mechanism of memory and learning, synaptic plasticity has been studied experimentally and theoretically $[18,19]$. Insights from these studies have led to the cybernetic system concept, in which the structure of the system is adaptively modified by the system itself [20,21]. Recently, researchers in network science have focused on the adaptivity of real-world networks. As reviewed in Refs. [5,6], many real-world networks are adaptive, with connections that continuously change according to the states of the nodes. We can classify previous studies on this subject among diverse fields into three groups, according to how the states of the nodes are described. In class I, the node has a discrete state. In particular, the state is binary, often represented by a spin. This description is universal, and most recent studies on adaptive networks, including those on opinion dynamics [22-27], epidemic spreading [28], game theory [29-32], and neural network of binary neurons [33], 
are categorized into this class. Class II is "phase," which represents the timing of periodic motion. Recent studies have revealed that the synaptic plasticity depends on the timing of neuronal activity [34-36], igniting the development of studies of spiking neural networks [37-49]. This class also includes the continuous state of a fine-grained discrete state (e.g., opinion dynamics which consider the spectrum of people's opinions [50,51]). Class III represents an "amount" of something, which is continuous and typically ranges in $[0, \infty)$. Our model can be categorized in this class, with the quantity being referred to as a "resource." Previous studies of reinforced random walks [52-54], reaction-diffusion dynamics [55], and gene regulation network [56,57] are categorized in this class. Another example is slime molds that form a network of tubes interconnecting the food sources. The tube thickness adaptively changes, depending on the flow of protoplasm [58].

The dynamics of real-world networks, which correspond to changes in the interactions in the systems, can be divided into two steps. The first step is a topological change, which creates new interactions or deletes existing interactions from the system. Little information is currently available on this coevolutionary elementary process in real-world networks, expect for a few studies on Twitter data [59]. The second step is a change in the link weights, which modifies the interaction strength. Although knowledge about this process is also limited, studies of the neural networks and autocatalytic chemical reaction systems can provide some clues. Based on these considerations, we have neglected the first step and considered only the second step in our model. The underlying topology $a_{i j}$ is assumed to be static. The weight $w_{i j}(t)$ of the existing links changes according to Eq. (2), which is similar to the law of mass action in chemical reactions or the Hebbian rule in neural networks. Interestingly, this simple equation can generate rich behaviors of the dynamical system with large degrees of freedom. Assuming a fixed underlying topology is justified if the topological change of the interactions is very slow, or if the mechanisms governing the two kinds of network dynamics are intrinsically different and activated in different periods. For example, in autocatalytic chemical reaction systems, the reaction rates of proteins (i.e., the link weights) can be drastically controlled by their own products, whereas the path of possible reactions (i.e., underlying network topology) is constant. In the neural networks, the synaptic plasticity of the network weights and synaptogenesis for the synaptic formation are believed to be regulated by different factors. The model could also be applied to economic and traffic networks. In business relationships, the resource is money and the interactions are trades between companies. A network based on Japanese economic trades was studied by a model similar to ours [60]. The potential application of this model to traffic networks, especially networks of traffic on the Internet, would be important for the understanding of the interplay between the network-reformation and the the PageRank, which is used to evaluate the number of page views on the web.

In Secs. III and IV, we have found that, under suitable conditions, the co-evolving dynamics lead to the organization of stationary power-law distributions of the resource and link weights. This finding is intuitively explained by the positive feedback between the resource amount and the inward weights.
As the resource is concentrated into nodes with thicker inward links, the link weights are strengthened by the concentrated resource. This scenario can provide another mechanism of the "rich-get-richer" law, which can be implemented by a dynamical system without knowing the global information over the network, such as the degree distribution required in the previous model [61].

The emergence of the power-law distribution of the resource has been numerically and theoretically studied, especially in the case of the regular random graph. We find two distinct regions in $(\kappa, D)$ space: the homogeneous resource distribution with a frozen state, and the power-law resource distribution with an unsteady microscopic state. There is no intermediate state between these extreme situations. Considering economic networks as an example, $\kappa$ controls the equal redistribution of money, similar to a tax; and $D$ controls the flow of money via trades. Our results imply that it is a very difficult task to avoid the extreme situations and realize a moderate state asymptotically by controlling these parameters.

We have studied the unsteady microscopic dynamics of the organized network with stationary distributions from the perspective of a deterministic dynamical system. This system exhibits multistability including many fixed points, limit cycles, and chaotic states. Despite these different microscopic behaviors, the stationary power-law distribution of the resource is robustly realized. For a small network, the system tends to be trapped at fixed points, implying the permanent oligopoly of the resource over the network. For a large network, the dynamical system almost always exhibits chaotic behavior, in which the current monopoly of the resource will lose its status and be replaced by the next winners. Microscopic dynamics with stationary power-law distributions are observed in real-world networks, such as city populations [62] and US airport networks [63]. Thus, the intrinsic interplay between the states of the nodes and the network reformation is essential for the dynamic aspects of the vicissitudes that are often observed in changing real-world networks.

\section{ACKNOWLEDGMENTS}

We thank N. Masuda, Hideki Takayasu, Misako Takayasu, Koutarou Tamura, and Naoya Fujiwara for fruitful discussions. This work was supported by JSPS KAKENHI Grants No. 24120708, No. 24740266, No. 25115719, and No. 26520206.

\section{APPENDIX: RELATIONSHIP BETWEEN SCALING EXPONENTS OF THE RESOURCE AND THE LINK WEIGHTS}

We derive the relationship between the exponents $\gamma_{x}, \gamma_{w}$ of the power-law distribution of the resource and the link weights, under two strong assumptions regarding the resource quantities at the adjacent nodes, $x_{i}$ and $x_{j}$.

First, we assume that there is no correlation between the resource quantities at adjacent nodes. According to Eq. (2), the link weights $w_{i j}$ will converge to $x_{i} x_{j}$. Thus, we approximate the weight by

$$
w_{i j}=x_{i} x_{j}
$$


The amounts of the resource, $x_{i}, x_{j}$, obey the power-law distribution with exponents $\gamma_{x}: P_{x}\left(x^{\prime}\right) \propto x^{\prime-\gamma_{x}}$. Therefore, the distribution of the link weight $w_{i j}$ can be derived according to Eq. (A1), for the two independent random variables $x_{i}$ and $x_{j}$. This problem requires that we transform random variables from two independent variables $X$ and $Y$ to new variables $W=X Y$ and $Z=Y$. The joint distribution $P_{X, Y}$ and $P_{W, Z}$ satisfies the following equation:

$$
\begin{aligned}
P_{W, Z}(w, z) d w d z & =P_{X}(x) P_{Y}(y) d x d y \\
& =P_{X}(w / z) P_{Y}(z)\left|\begin{array}{ll}
\frac{d x}{d w} & \frac{d x}{d z} \\
\frac{d y}{d w} & \frac{d y}{d z}
\end{array}\right| d w d z \\
& =P_{X}(w / z) P_{Y}(z) \frac{1}{z} d w d z .
\end{aligned}
$$

Therefore, the distribution $P_{W}(w)$ can be expressed as

$$
P_{W}(w)=\int P_{X}(w / z) P_{Y}(z) \frac{1}{z} d z
$$

Applying the result to the power-law distribution $P_{X}(x)=$ $P_{Y}(x) \propto x^{-\gamma_{x}}$, we obtain

$$
P_{W}(w) \propto \int\left(\frac{w}{z}\right)^{-\gamma_{x}} z^{-\gamma_{x}} \frac{1}{z} d z \propto w^{-\gamma_{x}} .
$$

In the above derivation, we assume that the range of $x$ is bounded by some cutoff. Therefore, $\gamma_{w}=\gamma_{x}$.

Next, we assume that the correlation between the amounts of resource at adjacent nodes equals 1 . In other words, $x_{i}=$ $x_{j}$. Thus, Eq. (A1) can be written as

$$
w_{i j}=x_{i} x_{j}=x_{i}^{2},
$$

and the weight distribution $P_{W}$ can be expressed by the resource distribution $P_{X}$. As a result,

$$
P_{W}(w)=\frac{1}{2 \sqrt{w}} P_{X}(\sqrt{w}) \propto w^{-\frac{\gamma_{x}+1}{2}} .
$$

Therefore, $\gamma_{w}=\frac{\gamma_{x}+1}{2}$.
[1] R. Albert and A. Barabasi, Rev. Mod. Phys. 74, 47 (2002).

[2] M. Newman, SIAM Rev. 45, 167 (2003).

[3] A. Barrat, M. Barthélemy, and A. Vespignani, Dynamical Processes on Complex Networks (Cambridge University Press, Cambridge, 2008).

[4] S. Boccaletti, V. Latora, Y. Moreno, M. Chavez, and D.-U. Hwang, Phys. Rep. 424, 175 (2006).

[5] T. Gross and B. Blasius, J. R. Soc. Interface 5, 259 (2008).

[6] H. Sayama, I. Pestov, J. Schmidt, B. J. Bush, C. Wong, J. Yamanoi, and T. Gross, Comput. Math. Appl. 65, 1645 (2013).

[7] T. Aoki and T. Aoyagi, Phys. Rev. Lett. 109, 208702 (2012).

[8] A. Vespignani, Nat. Phys. 8, 32 (2012).

[9] G. K. Zipf, Selective Studies and the Principle of Relative Frequency in Language (Harvard University Press, Cambridge, MA, 1932).

[10] G. Miller and E. Newman, Am. J. Psychol. 71, 209 (1958).

[11] H. Aoyama, W. Souma, Y. Nagahara, M. P. Okazaki, H. Takayasu, and M. Takayasu, Fractals 8, 293 (2000).

[12] A. Dragulescu and V. Yakovenko, Physica A 299, 213 (2001).

[13] X. Gabaix, Q. J. Econ. 114, 739 (1999).

[14] B. Huberman, P. Pirolli, J. Pitkow, and R. Lukose, Science 280 , 95 (1998).

[15] A. Clauset, C. Shalizi, and M. Newman, SIAM Rev. 51, 661 (2009).

[16] R. Pastor-Satorras and A. Vespignani, Phys. Rev. Lett. 86, 3200 (2001).

[17] D. O. Hebb, The Organization of Behavior (Wiley, New York, 1949).

[18] G. Shepherd, The Synaptic Organization of the Brain (Oxford University Press, Oxford, 2004).

[19] P. Dayan and L. Abbott, Theoretical Neuroscience: Computational and Mathematical Modeling of Neural Systems, Computational Neuroscience (MIT Press, Cambridge, MA, 2005).

[20] S. A. Kauffman, The Origins of Order: Self-organization and Selection in Evolution (Oxford University Press, Oxford, 1993).
[21] H. Haken, Synergetics: Introduction and Advanced Topics, Physics and Astronomy Online Library (Springer, Berlin, 2004).

[22] G. A. Böhme and T. Gross, Phys. Rev. E 83, 035101 (2011).

[23] P. Holme and M. E. J. Newman, Phys. Rev. E 74, 056108 (2006).

[24] C. Nardini, B. Kozma, and A. Barrat, Phys. Rev. Lett. 100, 158701 (2008).

[25] F. Vazquez, V. M. Eguíluz, and M. S. Miguel, Phys. Rev. Lett. 100, 108702 (2008).

[26] L.-X. Zhong, F. Ren, T. Qiu, J.-R. Xu, B.-H. Chen, and C.-F. Liu, Physica A 389, 2557 (2010).

[27] S. Mandrà, S. Fortunato, and C. Castellano, Phys. Rev. E 80, 056105 (2009).

[28] T. Gross, C. J. D. D’Lima, and B. Blasius, Phys. Rev. Lett. 96, 208701 (2006).

[29] J. M. Pacheco, A. Traulsen, and M. A. Nowak, Phys. Rev. Lett. 97, 258103 (2006).

[30] M. Perc and A. Szolnoki, BioSystems 99, 109 (2010).

[31] J. Poncela, J. Gómez-Gardeñes, A. Traulsen, and Y. Moreno, New J. Phys. 11, 083031 (2009).

[32] M. G. Zimmermann, V. M. Eguíluz, and M. San Miguel, Phys. Rev. E 69, 065102 (2004).

[33] S. Shinomoto, J. Phys. A: Math. Gen. 20, L1305 (1987).

[34] H. Markram, J. Lübke, M. Frotscher, and B. Sakmann, Science 275, 213 (1997).

[35] G.-Q. Bi and M.-M. Poo, J. Neurosci. 18, 10464 (1998).

[36] N. Caporale and Y. Dan, Annu. Rev. Neurosci. 31, 25 (2008).

[37] J. Karbowski and G. B. Ermentrout, Phys. Rev. E 65, 031902 (2002).

[38] P. Seliger, S. C. Young, and L. S. Tsimring, Phys. Rev. E 65, 041906 (2002).

[39] D. H. Zanette and A. S. Mikhailov, Europhys. Lett. 65, 465 (2004).

[40] N. Masuda and H. Kori, J. Comput. Neurosci. 22, 327 (2007).

[41] Y. L. Maistrenko, B. Lysyansky, C. Hauptmann, O. Burylko, and P. A. Tass, Phys. Rev. E 75, 066207 (2007).

[42] F. Sorrentino and E. Ott, Phys. Rev. Lett. 100, 114101 (2008). 
[43] H. Cateau, K. Kitano, and T. Fukai, Phys. Rev. E 77, 051909 (2008).

[44] M. Chen, Y. Shang, Y. Zou, and J. Kurths, Phys. Rev. E 77, 027101 (2008).

[45] T. Aoki and T. Aoyagi, Phys. Rev. Lett. 102, 034101 (2009).

[46] R. Gutiérrez, A. Amann, S. Assenza, J. Gómez-Gardeñes, V. Latora, and S. Boccaletti, Phys. Rev. Lett. 107, 234103 (2011).

[47] T. Aoki and T. Aoyagi, Phys. Rev. E 84, 066109 (2011).

[48] J. Ito and K. Kaneko, Phys. Rev. Lett. 88, 028701 (2001).

[49] J. Ito and K. Kaneko, Phys. Rev. E 67, 046226 (2003).

[50] F. Bagnoli, T. Carletti, D. Fanelli, A. Guarino, and A. Guazzini, Phys. Rev. E 76, 066105 (2007).

[51] B. Kozma and A. Barrat, Phys. Rev. E 77, 016102 (2008).

[52] B. Skyrms and R. Pemantle, Proc. Natl. Proc. Acad. Sci. USA 97, 9340 (2000).

[53] S.-W. Kim and J. D. Noh, Phys. Rev. Lett. 100, 118702 (2008).

[54] Q. Mei, J. Guo, and D. Radev, in Proceedings of the 16th ACM SIGKDD International Conference on Knowledge Discovery and Data Mining (ACM, New York, 2010), pp. 1009-1018.
[55] Q. Xuan, F. Du, H. Dong, L. Yu, and G. Chen, Phys. Rev. E 84, 036101 (2011)

[56] C. Furusawa and K. Kaneko, Phys. Rev. Lett. 90, 088102 (2003).

[57] C. Furusawa and K. Kaneko, Phys. Rev. Lett. 108, 208103 (2012).

[58] A. Tero, S. Takagi, T. Saigusa, K. Ito, D. P. Bebber, M. D. Fricker, K. Yumiki, R. Kobayashi, and T. Nakagaki, Science 327, 439 (2010).

[59] D. Antoniades and C. Dovrolis, arXiv:1309.6001 (2013).

[60] K. Tamura, W. Miura, M. Takayasu, H. Takayasu, S. Kitajima, and H. Goto, Int. J. Mod. Phys. Conf. Ser. 16, 93 (2012).

[61] A. Barabási and R. Albert, Science 286, 509 (1999).

[62] M. Batty, Nature (London) 444, 592 (2006).

[63] A. Gautreau, A. Barrat, and M. Barthélemy, Proc. Natl. Proc. Acad. Sci. USA 106, 8847 (2009).

[64] See Supplemental Material at http://link.aps.org/supplemental/ 10.1103/PhysRevE.91.012908 for a movie showing a typical behavior of the model. 